\title{
The CFS-PML for 2D Auxiliary Differential Equation FDTD Method Using Associated Hermite Orthogonal Functions
}

\author{
Feng Jiang, ${ }^{1}$ Xiao-Ping Miao, ${ }^{1}$ Feng Lu, ${ }^{2}$ Li-Yuan Su, ${ }^{3}$ and Yao $\mathrm{Ma}^{3}$ \\ ${ }^{1}$ College of Defense Engineering, PLA University of Science and Technology, Nanjing 210007, China \\ ${ }^{2}$ Jiangsu Regulatory Bureau of Nuclear and Radiation Safety, Nanjing 210019, China \\ ${ }^{3}$ National Key Laboratory on Electromagnetic Environmental Effects and Electro-Optical Engineering, \\ PLA University of Science and Technology, Nanjing 210007, China \\ Correspondence should be addressed to Feng Lu; fenglu76@163.com
}

Received 25 October 2016; Revised 18 February 2017; Accepted 26 March 2017; Published 18 May 2017

Academic Editor: Sotirios K. Goudos

Copyright (C) 2017 Feng Jiang et al. This is an open access article distributed under the Creative Commons Attribution License, which permits unrestricted use, distribution, and reproduction in any medium, provided the original work is properly cited.

\begin{abstract}
The complex frequency shifted (CFS) perfectly matched layer (PML) is proposed for the two-dimensional auxiliary differential equation (ADE) finite-difference time-domain (FDTD) method combined with Associated Hermite (AH) orthogonal functions. According to the property of constitutive parameters of CFS-PML (CPML) absorbing boundary conditions (ABCs), the auxiliary differential variables are introduced. And one relationship between field components and auxiliary differential variables is derived. Substituting auxiliary differential variables into CPML ABCs, the other relationship between field components and auxiliary differential variables is derived. Then the matrix equations are obtained, which can be unified with Berenger's PML (BPML) and free space. The electric field expansion coefficients can thus be obtained, respectively. In order to validate the efficiency of the proposed method, one example of wave propagation in two-dimensional free space is calculated using BPML, UPML, and CPML. Moreover, the absorbing effectiveness of the BPML, UPML, and CPML is discussed in a two-dimensional (2D) case, and the numerical simulations verify the accuracy and efficiency of the proposed method.
\end{abstract}

\section{Introduction}

In the conventional finite-difference time-domain (FDTD) method $[1,2]$, the time step is constrained by the CourantFriedrichs-Lewy (CFL) stability condition. When fine structures such as thin material and slot are simulated, more computer memory and computation time are required. In order to eliminate the CFL stability condition, some unconditionally stable FDTD methods have been developed such as alternating-direction implicit (ADI) method [3], CrankNicolson method [4], locally one-dimensional method [5], and Weighted Laguerre Polynomials (WLP) FDTD method [6]. Recently, the Associated Hermite (AH) FDTD methods to model wave propagation have been introduced in $[7,8]$.

The perfectly matched layer (PML) absorbing boundary conditions (ABCs) introduced by Berenger [9] have been widely used for truncating FDTD domains. Some PML variants have been proposed to improve the absorbing effectiveness for various electromagnetic waves, like modified PML
(MPML), uniaxial anisotropic PML (UPML), generalized PML (GPML), and so forth. Recently, UPML-ABC for AHFDTD method [10] is used in conductive medium. According to Kuzuoglu and Mittra in [11], the complex frequency shifted (CFS) PML $[12,13]$ is the most accurate in all the PML. The frequency-domain coordinate-stretching variable of CFSPML (CPML) has three adjustable variables, while there are two adjustable variables for UPML-ABC. The reflection error of CPML can be greatly reduced from adjusting variables, and it has been implemented in the Cartesian coordinate, periodic structures, cylindrical coordinates, dispersive materials, WLP-FDTD, and so on.

In this paper, a 2D CPML for auxiliary differential equation (ADE) FDTD method using AH orthogonal functions is proposed. It is shown that the constitutive parameters of CPML are complicated, and if the method in [7] is used directly here, the second derivative field components would be involved and the final matrix equations will also be complex. In order to get a simple and easy formula, the 
auxiliary differential variables are introduced. Based on the $\mathrm{ADE}$ technique $[14,15]$ and Galerkin temporal testing procedure, one relationship between field components and auxiliary differential variables is derived. According to auxiliary differential variables and Maxwell's equations of CPML, the other relationship between field components and auxiliary differential variables is derived. Then the formulations of all orders of $\mathrm{AH}$ functions are obtained to calculate the magnetic field expansion coefficients. According to [7], the matrix equations of CPML, Berenger's PML (BPML), and free space can be unified. At last, the electric field expansion coefficients can also be obtained, respectively. To validate the efficiency of the proposed method, a 2D case is calculated. And the efficiency of the proposed method is verified through the comparison with BPML and UPML ABCs.

\section{Formulation}

With free space, the frequency-domain Maxwell's equations of CPML for $2 \mathrm{D} \mathrm{TE}_{\mathrm{z}}$ model case are

$$
\begin{aligned}
j \omega \varepsilon_{0} E_{x} & =\frac{1}{R_{y}} \frac{\partial H_{z}}{\partial y}, \\
j \omega \varepsilon_{0} E_{y} & =-\frac{1}{R_{x}} \frac{\partial H_{z}}{\partial x}, \\
j \omega \mu_{0} H_{z x} & =-\frac{1}{R_{x}} \frac{\partial E_{y}}{\partial x}, \\
j \omega \mu_{0} H_{z y} & =\frac{1}{R_{y}} \frac{\partial E_{x}}{\partial y},
\end{aligned}
$$

where $\varepsilon_{0}$ is the electric permittivity and $\mu_{0}$ is the magnetic permeability of free space; $R_{\Phi}=\kappa_{\Phi}+\sigma_{\Phi} /\left(\alpha_{\Phi}+j \omega \varepsilon_{0}\right), \Phi=$ $x, y$. For CPML, $\alpha_{\Phi}$ and $\sigma_{\Phi}$ are assumed to be positive real and $\kappa_{\Phi}$ is real and $\geq 1$.

An orthonormal set of basis functions $\left\{\varphi_{0}, \varphi_{1}, \varphi_{2}, \varphi_{3}, \ldots\right\}$ can be defined as

$$
\phi_{n}(\tilde{t})=\left(2^{n} n ! \pi^{1 / 2}\right)^{-1 / 2} e^{-\widetilde{t}^{2} / 2} H_{n}(\widetilde{t}),
$$

where $H_{n}(\widetilde{t})=(-1)^{n} e^{\widetilde{t}^{2}}\left(d^{n} / d t^{n}\right)\left(e^{-\widetilde{t}^{2}}\right)$ are Hermite polynomials, $\widetilde{t}=\left(t-T_{f}\right) / \gamma, T_{f}$ is a time-translating parameter, and $\gamma$ is a time-scaling parameter. Choosing a finite $Q$ order of basis functions [7] and proper parameters for $T_{f}$ and $\gamma$, and then using these transformed basis functions, the causal field components, taking $u$ for example, can be expanded as

$$
u(r, t)=\sum_{n=0}^{Q} u_{n}(r) \bar{\phi}_{n}(\tilde{t}) .
$$

The time derivative of the $n$th order $\mathrm{AH}$ function is

$$
\begin{aligned}
& \frac{d \bar{\phi}_{n}(\tilde{t})}{d t} \quad(n=0), \\
& \quad= \begin{cases}-\frac{1}{\gamma} \sqrt{\frac{1}{2}} \bar{\phi}_{1}(\tilde{t}), & (n \geq 1) . \\
\frac{1}{\gamma} \sqrt{\frac{n}{2}} \bar{\phi}_{n-1}(\tilde{t})-\frac{1}{\gamma} \sqrt{\frac{n+1}{2}} \bar{\phi}_{n+1}(\tilde{t}), & \end{cases}
\end{aligned}
$$

According to (4), the first derivative of field components $u(r, t)$ with respect to $t$ is [7]

$$
\begin{aligned}
& \frac{\partial}{\partial t} u(r, t) \\
& \quad=\frac{1}{\gamma} \sum_{n=0}^{Q}\left(u_{n+1}(r) \sqrt{\frac{n+1}{2}}-u_{n-1}(r) \sqrt{\frac{n}{2}}\right) \bar{\phi}_{n}(\tilde{t}),
\end{aligned}
$$

where $u_{-1}(r)=0$.

Using ADE scheme $[13,14]$, four auxiliary differential variables are introduced:

$$
\begin{aligned}
\psi_{E x} & =\frac{j \omega E_{x}}{\alpha_{y}+j \omega \varepsilon_{0}}, \\
\psi_{E y} & =\frac{j \omega E_{y}}{\alpha_{x}+j \omega \varepsilon_{0}}, \\
\psi_{H z x} & =\frac{j \omega H_{z x}}{\alpha_{x}+j \omega \varepsilon_{0}}, \\
\psi_{H z y} & =\frac{j \omega H_{z y}}{\alpha_{y}+j \omega \varepsilon_{0}} .
\end{aligned}
$$

With the transition relationship from frequency domain to time domain, (6) can be written as

$$
\alpha_{y} \psi_{E x}+\varepsilon_{0} \frac{\partial \psi_{E x}}{\partial t}=\frac{\partial E_{x}}{\partial t} .
$$

Applying (3) and (5) to (10), the field functions in (10) can be expanded by

$$
\begin{aligned}
& \left(\alpha_{y} \psi_{E x}^{p}(r)\right. \\
& \left.+\varepsilon_{0} \frac{1}{\gamma} \sum_{n=0}^{Q}\left(\psi_{E x}^{n+1}(r) \sqrt{\frac{n+1}{2}}-\psi_{E x}^{n-1}(r) \sqrt{\frac{n}{2}}\right)\right) \\
& \cdot \bar{\phi}_{n}(\tilde{t})=\frac{1}{\gamma} \sum_{n=0}^{Q}\left(E_{x}^{n+1}(r) \sqrt{\frac{n+1}{2}}-E_{x}^{n-1}(r) \sqrt{\frac{n}{2}}\right) \\
& \cdot \bar{\phi}_{n}(\tilde{t}) .
\end{aligned}
$$

Multiplying both sides by $\bar{\phi}_{n}(\widetilde{t})$ and integrating over $(-\infty, \infty)$, we get

$$
\begin{gathered}
\sqrt{\frac{n+1}{2}} \frac{\varepsilon_{0}}{\gamma} \psi_{E x}^{q+1}(r)+\alpha_{y} \psi_{E x}^{q}(r)-\sqrt{\frac{n}{2}} \frac{\varepsilon_{0}}{\gamma} \psi_{E x}^{q-1}(r) \\
=\sqrt{\frac{n+1}{2}} \frac{1}{\gamma} E_{x}^{q+1}(r)-\sqrt{\frac{n}{2}} \frac{1}{\gamma} E_{x}^{q-1}(r) .
\end{gathered}
$$


Applying the same above procedure with (7)-(9), we have

$$
\begin{aligned}
& \sqrt{\frac{n+1}{2}} \frac{\varepsilon_{0}}{\gamma} \psi_{H z y}^{q+1}(r)+\alpha_{y} \psi_{H z y}^{q}(r)-\sqrt{\frac{n}{2}} \frac{\varepsilon_{0}}{\gamma} \psi_{H z y}^{q-1}(r) \\
& =\sqrt{\frac{n+1}{2}} \frac{1}{\gamma} H_{z y}^{q+1}(r)-\sqrt{\frac{n}{2}} \frac{1}{\gamma} H_{z y}^{q-1}(r) .
\end{aligned}
$$

$$
\begin{aligned}
& =\sqrt{\frac{n+1}{2}} \frac{1}{\gamma} E_{y}^{q+1}(r)-\sqrt{\frac{n}{2}} \frac{1}{\gamma} E_{y}^{q-1}(r), \\
& \sqrt{\frac{n+1}{2}} \frac{\varepsilon_{0}}{\gamma} \psi_{H z x}^{q+1}(r)+\alpha_{x} \psi_{H z x}^{q}(r)-\sqrt{\frac{n}{2}} \frac{\varepsilon_{0}}{\gamma} \psi_{H z x}^{q-1}(r) \\
& =\sqrt{\frac{n+1}{2}} \frac{1}{\gamma} H_{z x}^{q+1}(r)-\sqrt{\frac{n}{2}} \frac{1}{\gamma} H_{z x}^{q-1}(r),
\end{aligned}
$$

$$
\begin{aligned}
& {[\xi]_{\Phi}=\left[\begin{array}{cccc}
\alpha_{\Phi} & \sqrt{\frac{1}{2} \frac{\varepsilon_{0}}{\gamma}} & & \\
-\sqrt{\frac{1}{2} \frac{\varepsilon_{0}}{\gamma}} & \alpha_{\Phi} & \sqrt{\frac{1+1}{2} \frac{\varepsilon_{0}}{\gamma}} & \\
\ddots & -\sqrt{\frac{Q-2}{2} \frac{\varepsilon_{0}}{\gamma}} & \alpha_{\Phi} & \sqrt{\frac{Q-2+1}{2}} \frac{\varepsilon_{0}}{\gamma} \\
& -\sqrt{\frac{Q-1}{2} \frac{\varepsilon_{0}}{\gamma}} & \alpha_{\Phi}
\end{array}\right] \quad(\Phi=x, y)}
\end{aligned}
$$

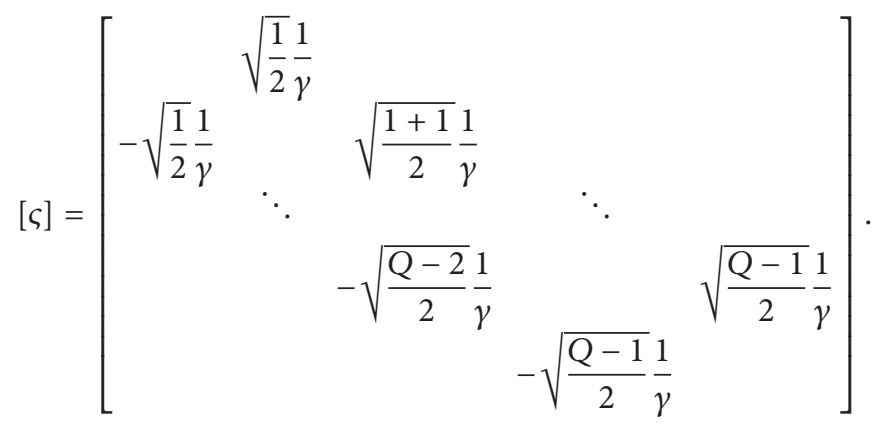

Equation (14) can be rewritten in a new matrix form:

$$
\begin{aligned}
\psi_{E x} & =[\xi]_{y}^{-1}[\varsigma] E_{x}, \\
\psi_{E y} & =[\xi]_{x}^{-1}[\varsigma] E_{y}, \\
\psi_{H z x} & =[\xi]_{x}^{-1}[\varsigma] H_{z x}, \\
\psi_{H z y} & =[\xi]_{y}^{-1}[\varsigma] H_{z y} .
\end{aligned}
$$

Applying (6)-(9) to (1) and transferring from frequency domain to time domain, we have

$$
\begin{aligned}
& \varepsilon_{0}\left(\kappa_{y} \frac{\partial E_{x}}{\partial t}+\sigma_{y} \psi_{E x}\right)=\frac{\partial H_{z}}{\partial y}, \\
& \varepsilon_{0}\left(\kappa_{x} \frac{\partial E_{y}}{\partial t}+\sigma_{x} \psi_{E y}\right)=-\frac{\partial H_{z}}{\partial x},
\end{aligned}
$$

Similar to $[7,8]$, we can rewrite (12)-(13) in a matrix form:

$$
\begin{aligned}
{[\xi]_{y} \psi_{E x} } & =[\varsigma] E_{x}, \\
{[\xi]_{x} \psi_{E y} } & =[\varsigma] E_{y}, \\
{[\xi]_{x} \psi_{H z x} } & =[\varsigma] H_{z x}, \\
{[\xi]_{y} \psi_{H z y} } & =[\varsigma] H_{z y},
\end{aligned}
$$

where 


$$
\begin{aligned}
&=-\frac{\gamma}{\mu_{0}}[\beta] \frac{\partial E_{y}^{q}(r)}{\partial x}, \\
& \kappa_{j+0.5}[\alpha] H_{z y}^{q}(r)+\gamma \sigma_{j+0.5}[\beta] \psi_{H z y}^{q}(r) \\
&=\frac{\gamma}{\mu_{0}}[\beta] \frac{\partial E_{x}^{q}(r)}{\partial y},
\end{aligned}
$$

where

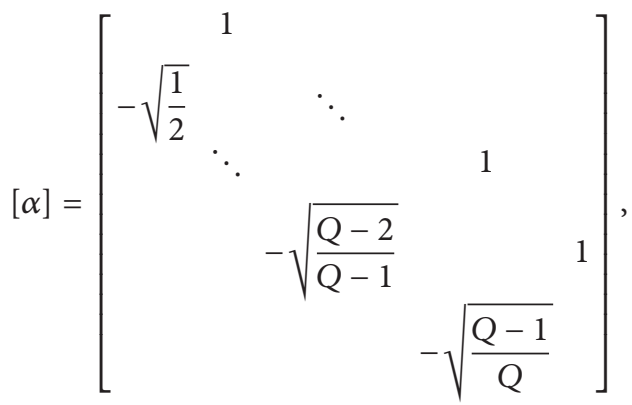

$$
\begin{aligned}
& {[\beta]=\left[\begin{array}{cccc}
\sqrt{\frac{2}{1}} & & & \\
& \sqrt{\frac{2}{2}} & & \\
& \ddots & \\
& & \sqrt{\frac{2}{Q-1}} & \\
& & & \sqrt{\frac{2}{Q}}
\end{array}\right] .}
\end{aligned}
$$

Using central difference scheme, substituting (16) into (18), grafting (18), and using $H_{z}=H_{z x}+H_{z y}$, we have

$$
\begin{aligned}
& {\left[E_{x}\right]_{i+0.5, j}=\left.\bar{C}_{y}^{E}\right|_{i, j}[\eta]_{j}^{-1}[\beta]} \\
& \cdot\left(\left[H_{z}\right]_{i+0.5, j+0.5}-\left[H_{z}\right]_{i+0.5, j-0.5}\right) \\
& {\left[E_{y}\right]_{i, j+0.5}=-\left.\bar{C}_{x}^{E}\right|_{i, j}[\eta]_{i}^{-1}[\beta]} \\
& \cdot\left(\left[H_{z}\right]_{i+0.5, j+0.5}-\left[H_{z}\right]_{i-0.5, j+0.5}\right) \\
& {\left[H_{z}\right]_{i+0.5, j+0.5}=\left.\bar{C}_{y}^{H}\right|_{i, j}[\eta]_{j+0.5}^{-1}[\beta]} \\
& \cdot\left(\left[E_{x}\right]_{i+0.5, j+1}-\left[E_{x}\right]_{i+0.5, j}\right)-\left.\bar{C}_{x}^{H}\right|_{i, j}[\eta]_{i+0.5}^{-1}[\beta] \\
& \cdot\left(\left[E_{y}\right]_{i+1, j+0.5}-\left[E_{y}\right]_{i, j+0.5}\right)
\end{aligned}
$$

where $[\eta]_{\Phi}=\kappa_{\Phi}[\alpha]+\gamma \sigma_{\Phi}[\beta][\xi]_{\Phi}^{-1}[\varsigma](\Phi=x, y),\left.\bar{C}_{x}^{E}\right|_{i, j}=$ $\gamma / \varepsilon_{0} \Delta \bar{x}_{i},\left.\bar{C}_{y}^{E}\right|_{i, j}=\gamma / \varepsilon_{0} \Delta \bar{y}_{j},\left.\bar{C}_{x}^{H}\right|_{i, j}=\gamma / \mu_{0} \Delta x_{i},\left.\bar{C}_{y}^{H}\right|_{i, j}=$ $\gamma / \mu_{0} \Delta y_{j}$, and $\Delta x_{i}$ and $\Delta y_{j}$ are the lengths of the cell edge where the electric fields are located. $\Delta \bar{x}_{i}$ and $\Delta \bar{y}_{j}$ are the distances between the center nodes where the magnetic fields

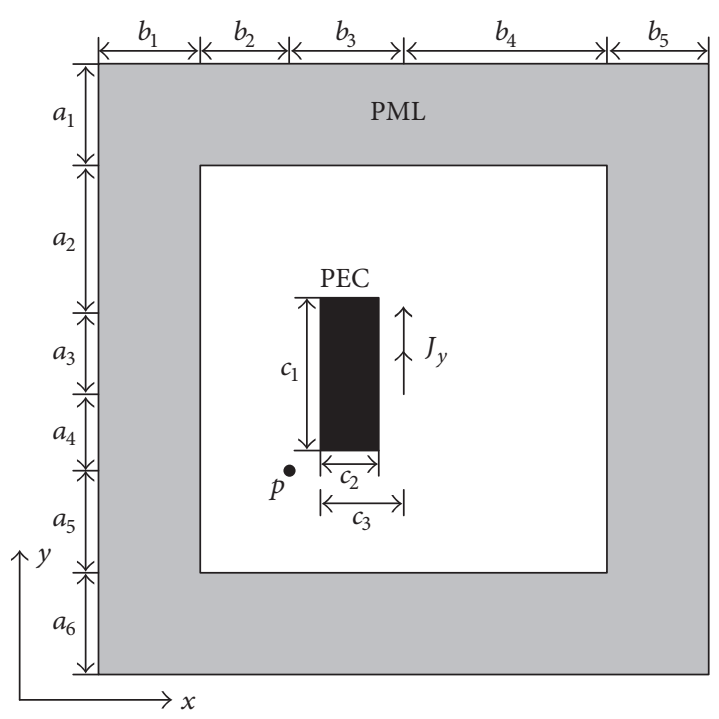

Figure 1: Computational domain.

are located. For the above $(i, j)$ subscripts for $C_{x}$ and $C_{y}$ in $(20)-(22),(i, j)$ is not a real position but an array index of each field variable, as shown in Figure 1 of [16].

In order to form the matrix equations only containing magnetic field, we apply (20)-(21) to (22):

$$
\begin{gathered}
b_{l}\left[H_{z}\right]_{i-0.5, j+0.5}+b_{r}\left[H_{z}\right]_{i+1.5, j+0.5}+a\left[H_{z}\right]_{i+0.5, j+0.5} \\
+b_{u}\left[H_{z}\right]_{i+0.5, j+1.5}+b_{d}\left[H_{z}\right]_{i+0.5, j-0.5}=0,
\end{gathered}
$$

where

$$
\begin{aligned}
& b_{l}=\left.\left.\bar{C}_{x}^{H}\right|_{i, j} \bar{C}_{x}^{E}\right|_{i, j}[\eta]_{i+0.5}^{-1}[\beta][\eta]_{i}^{-1}[\beta], \\
& b_{r}=\left.\left.\bar{C}_{x}^{H}\right|_{i, j} \bar{C}_{x}^{E}\right|_{i+1, j}[\eta]_{i+0.5}^{-1}[\beta][\eta]_{i+1}^{-1}[\beta], \\
& b_{u}=\left.\left.\bar{C}_{y}^{H}\right|_{i, j} \bar{C}_{y}^{E}\right|_{i, j+1}[\eta]_{j+0.5}^{-1}[\beta][\eta]_{j+1}^{-1}[\beta], \\
& b_{d}=\left.\left.\bar{C}_{y}^{H}\right|_{i, j} \bar{C}_{y}^{E}\right|_{i, j}[\eta]_{j+0.5}^{-1}[\beta][\eta]_{j}^{-1}[\beta], \\
& a=-\left(b_{u}+b_{d}+b_{l}+b_{r}+I\right) .
\end{aligned}
$$

$I$ is unit matrix.

From (23), only magnetic field vector variables remained. And it can also be found that each magnetic field variable is related to the four adjacent magnetic fields. If $R=1 \mathrm{in}$ (1), the final matrix equations of CPML will degrade to the matrix equations of free space in AH-FDTD method. If $\kappa=1$ and $\alpha_{\Phi}=0$ in (1), the final matrix equations of CPML will degrade to the matrix equations of BPML in AH-FDTD method. So the matrix equations of CPML and BPML and free space can be unified. And this greatly facilitates the programming. Finally, a banded sparse coefficient matrix is obtained like [7], which contains all the points in free space and CPML ABC. And the paralleling-in-order solution scheme in [8] is applied to indirectly but efficiently calculate all of the expansion 
coefficients. If all of the expansion coefficients of the magnetic field are calculated, the expansion coefficients of the electric field can be obtained from (20)-(21). Finally, $E_{x}(r, t), E_{y}(r, t)$, and $H_{z}(r, t)$ can be reconstructed by using (3).

\section{Numerical Demonstration}

In order to validate the efficiency of the presented method, wave scatter from a PEC medium is simulated. The BPML, UPML, and CPML ABCs are used to truncate the FDTD domains. The PML constitutive parameters are scaled using $m$ th order polynomial scaling [17]:

$$
\begin{aligned}
\sigma\left(\rho_{\Phi}\right) & =\sigma_{\Phi, \max }\left(\frac{\rho_{\Phi}}{D_{\Phi}}\right)^{m} \quad(\Phi=x, y), \\
\sigma_{\mathrm{opt}} & =\frac{(m+1)}{150 \pi \Delta \rho}, \\
\kappa\left(\rho_{\Phi}\right) & =1+\left(\kappa_{\Phi, \max }-1\right)\left(\frac{\rho_{\Phi}}{D_{\Phi}}\right)^{m} \quad(\Phi=x, y),
\end{aligned}
$$

where $\rho$ indicates the distance from the dispersive mediumPML interface into the PML, $D$ is the depth of the PML, and $m=4$ is the order of polynomial.

As illustrated in Figure 1, the computational domain is truncated by 10 additional PML in $x$-direction and $y$ direction, respectively. And we choose $b 1=b 5=a 1=a 6=$ $10 \mathrm{~mm}, a 2=12 \mathrm{~mm}, a 3=6 \mathrm{~mm}, a 4=10 \mathrm{~mm}, a 5=2 \mathrm{~mm}, b 2=$ $2 \mathrm{~mm}, b 3=13 \mathrm{~mm}$, and $b 4=15 \mathrm{~mm}$ (in Figure 1), which results in a $50 \times 50$ cells lattice with $\Delta x=\Delta y=1 \mathrm{~mm}$. For the PEC rectangle's scatter, we choose $c 1=16 \mathrm{~mm}, c 2=8 \mathrm{~mm}$, and $c 3$ $=10 \mathrm{~mm}$.

A sinusoidal-modulated Gaussian pulse is chosen as the $y$-direction excitation source:

$$
J_{y}(t)=e^{-\left(t-t_{c}\right)^{2} / t_{d}^{2}} \sin \left(2 \pi f_{c}\left(t-t_{c}\right)\right),
$$

where $t_{d}=1 /\left(2 f_{c}\right), t_{c}=3 t_{d}$, and $f_{c}=0.8 \mathrm{GHz}$. The time duration of interest for the analyzed fields is chosen as $T_{s}=50 \mathrm{~ns}$ and the bandwidth is limited up to the frequency $W_{s}=3 \mathrm{GHz}$. We choose the order $Q=300$ and the range of $\gamma=1.3 \times 10^{-9}$ from the condition above. The time-translating parameter $T_{f}=25 \mathrm{~ns}$.

In order to compare the performance of the proposed CPML with that of BPML, a reference solution was also simulated with no reflection coming from the boundary. And the same mesh is extended by 100 cells in $x$-direction and $y$ direction, leading to a $250 \times 250$ cells lattice. And the reference field is calculated using AH-FDTD method. The reflection error is calculated as follows:

$$
R_{\mathrm{dB}}=20 \log _{10}\left(\frac{\left|E_{x}^{B}(t)-E_{x}^{T}(t)\right|}{\max \left|E_{x}^{B}(t)\right|}\right)
$$

where $E_{x}^{T}(t)$ is the field computed in the test domain and $E_{x}^{B}(t)$ is the reference field.

In Figure 2, the waveform of electric field at points $P$ is graphed by reference field, BPML, UPML, and CPML. And

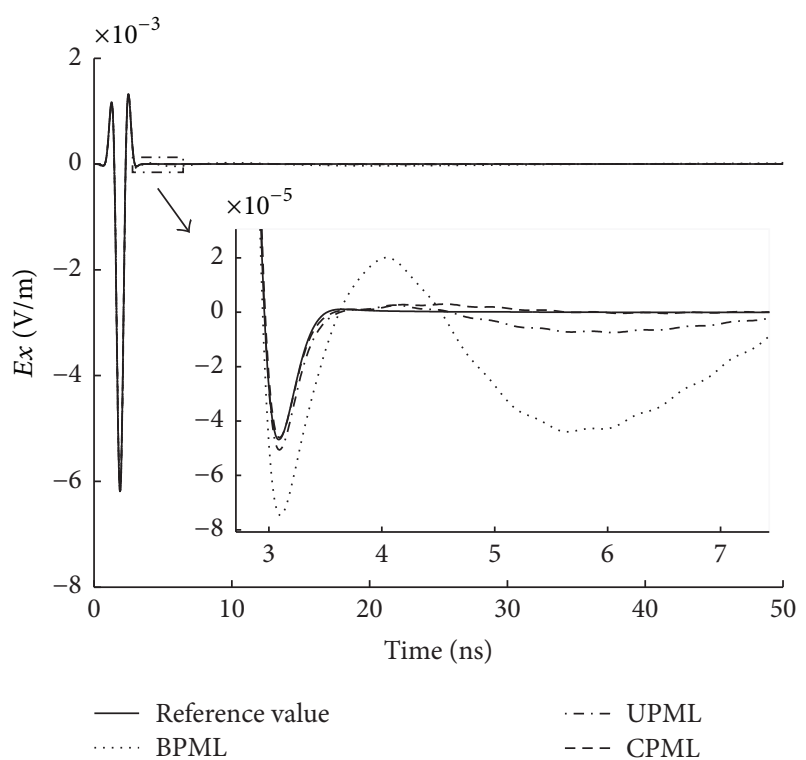

FIgURE 2: Transient electric field of $x$ component at the observation point.

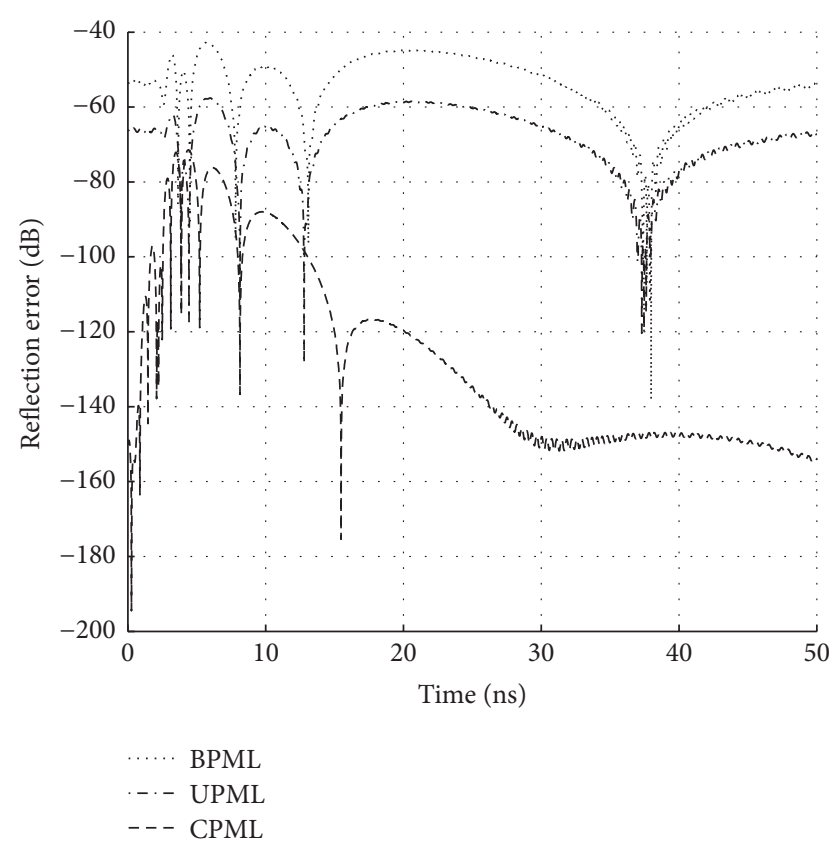

FIGURE 3: Reflection error in the electric field intensity relative to the field's maximum amplitude versus time for BPML (with $D=10$ cells, $\sigma_{\max } / \sigma_{\mathrm{opt}}=1$, and $m=4$ ), UPML (with $D=10$ cells, $\kappa_{\max }=22$, $\sigma_{\max } / \sigma_{\text {opt }}=1.2$, and $m=4$ ), and CPML (with $D=10$ cells, $\alpha=0.003$, $\kappa_{\max }=72, \sigma_{\max } / \sigma_{\text {opt }}=1.12$, and $m=4$ ) at point $p$.

it should be pointed out that parameters with different PML are different. Using (27), the reflection error is computed at the measurement point $P$. In Figure 3, it is noted that the maximum relative error is $-43 \mathrm{~dB},-58 \mathrm{~dB}$, and $-70 \mathrm{~dB}$ for the BPML, UPML, and CPML, respectively. And it is obvious that, with the CFS factor, the CPML is superior to 
TABLE 1: Comparison of CPU resource for various methods.

\begin{tabular}{lccc}
\hline Methods & $\Delta t(\mathrm{ps})$ & Memory $(\mathrm{Mb})$ & CPU time $(\mathrm{s})$ \\
\hline Conventional FDTD & 2.33 & 2.6 & 183 \\
UPML of [10] & 23.3 & 150.2 & 2.1 \\
WLP-FDTD & 23.3 & 39.5 & 8.1 \\
CPML of proposed method & 23.3 & 150.2 & 2.1 \\
\hline
\end{tabular}

BPML and UPML [10]. In Figures 2 and 3, the computation time is much higher than the time-support of source wave. And late-time reflections of CPML are much lower than those of UPML and BPML. The CFL stability condition of this model is $\Delta t \leq 2.33$ ps. The time step size for the proposed method is 23.3 ps. In Table 1 , comparison of CPU resource for four methods is presented. In order to guarantee computation accuracy, the CPML $[11,18]$ is also adopted in WLP-FDTD and conventional FDTD method. The total memory storage for the proposed method is increased to 150.2 Mb, about 57.8 times of the conventional FDTD method and 3.8 times of WLP-FDTD method, while the total CPU time for the proposed method can be reduced to about $1.1 \%$ of the conventional FDTD method and $25.9 \%$ of WLP-FDTD method.

\section{Conclusion}

Using ADE scheme, the CPML for 2D AH-FDTD method has been presented in this paper. This method is free from CFL stability condition for it has eliminated the time variable in calculation. The final matrix equations of CPML and BPML and free space can be unified. By solving the banded sparse coefficient matrix, magnetic field expansion coefficients of all orders of AH functions can be obtained. Then the electric field expansion coefficients can also be obtained, respectively. Numerical results show that this implementation is very effective in absorbing the electromagnetic waves, which means that the proposed method can save more computation time and computer memory.

\section{Conflicts of Interest}

The authors declare that there are no conflicts of interest regarding the publication of this paper.

\section{References}

[1] K. S. Yee, "Numerical solution of initial boundary value problems involving Maxwell equations in isotropic meidia," IEEE Trans. on Antennas and Propagat, vol. 14, no. 3, pp. 302-307, 1966.

[2] R. Xiong, B. Chen, Z. Cai, and Q. Chen, "A numerically efficient method for the FDTD analysis of the shielding effectiveness of large shielding enclosures with thin-slots," International Journal of Applied Electromagnetics and Mechanics, vol. 40, no. 4, pp. 251-258, 2012.

[3] F. Zheng and Z. Chen, "Numerical dispersion analysis of the unconditionally stable 3-D ADI-FDTD method," IEEE
Transactions on Microwave Theory and Techniques, vol. 49, no. 5, pp. 1006-1009, 2001.

[4] Y. Yang, R. S. Chen, and E. K. N. Yung, "The unconditionally stable crank-nicolson FDTD method for three-dimensional maxwell's equations," Microwave and Optical Technology Letters, vol. 48, no. 8, pp. 1619-1622, 2006.

[5] O. Ramadan, "Improved and efficient unconditionally stable complex-envelope frequency-dependent FDTD formulations based on the implicit locally one-dimensional scheme," Journal of Electromagnetic Waves and Applications, vol. 28, no. 3, pp. 334-345, 2014.

[6] F. Lu, Y. Ma, R. Xiong, Y.-T. Duan, and L.-Y. Su, "UPML$\mathrm{ABC}$ of dispersive materials for the unconditionally stable 2-D WLP-FDTD method," International Journal of Applied Electromagnetics and Mechanics, vol. 48, no. 4, pp. 447-457, 2015.

[7] Z.-Y. Huang, L.-H. Shi, B. Chen, and Y.-H. Zhou, "A new unconditionally stable scheme for FDTD method using associated Hermite orthogonal functions," IEEE Transactions on Antennas and Propagation, vol. 62, no. 9, pp. 4804-4809, 2014.

[8] H. Zheng-Yu, S. Li-Hua, Z. Ying-Hui, and C. Bin, "AnImproved paralleling-in-order solving scheme for AH-FDTD method using eigenvalue transformation," IEEE Transactions on Antennas and Propagation, vol. 63, no. 5, pp. 2135-2140, 2015.

[9] J.-P. Berenger, "A perfectly matched layer for the absorption of electromagnetic waves," Journal of Computational Physics, vol. 114, no. 2, pp. 185-200, 1994.

[10] Z.-Y. Huang, L.-H. Shi, Y. Zhou, and B. Chen, "UPML-ABC and TF/SF boundary for unconditionally stable AH-FDTD method in conductive medium," Electronics Letters, vol. 51, no. 21, pp. 1654-1656, 2015.

[11] M. Kuzuoglu and R. Mittra, "Frequency dependence of the constitutive parameters of causal perfectly matched anisotropie absorbers," IEEE Microwave and Guided Wave Letters, vol. 6, no. 12, pp. 447-449, 1996.

[12] J. Liu, G. Wan, J. Zhang, and X. Xi, "An effective CFS-PML implementation for cylindrical coordinate FDTD method," IEEE Microwave and Wireless Components Letters, vol. 22, no. 6, pp. 300-302, 2012.

[13] X. Xi, Y. Fang, J. Liu, and Z. Zhu, "An effective CFS-PML implementation for 2-D WLP-FDTD method," IEICE Electronics Express, vol. 12, no. 7, pp. 1-9, 2015.

[14] W.-J. Chen, W. Shao, H. Chen, and B.-Z. Wang, "Nearly PML for ADE-WLP-FDTD modeling in two-dimensional dispersive media," IEEE Microwave and Wireless Components Letters, vol. 24, no. 2, pp. 75-77, 2014.

[15] W.-J. Chen, W. Shao, and B.-Z. Wang, "ADE-laguerre-FDTD method for wave propagation in general dispersive materials," IEEE Microwave and Wireless Components Letters, vol. 23, no. 5, pp. 228-230, 2013.

[16] Y.-S. Chung, T. K. Sarkar, B. H. Jung, and M. Salazar-Palma, “An unconditionally stable scheme for the finite-difference timedomain method," IEEE Transactions on Microwave Theory and Techniques, vol. 51, no. 3, pp. 697-704, 2003.

[17] S. D. Gedney, "The perfectly matched layer absorbing medium," in Advances in Computational Electrodynamics: The Finite Difference Time Domain, A. Taflove, Ed., pp. 263-340, Artech House, Boston, Mass, USA, 1998.

[18] Y. Ma, S.-W. Chen, F. Lu, L.-Y. Su, and Y.-T. Duan, "The CFSPML for 2-D WLP-FDTD method of dispersive materials," International Journal of Applied Electromagnetics and Mechanics, vol. 51, no. 4, pp. 349-361, 2016. 


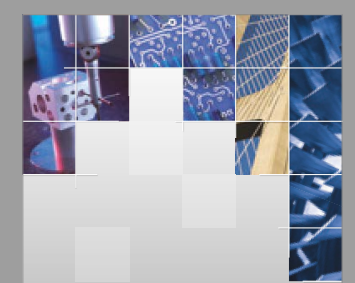

\section{Enfincering}
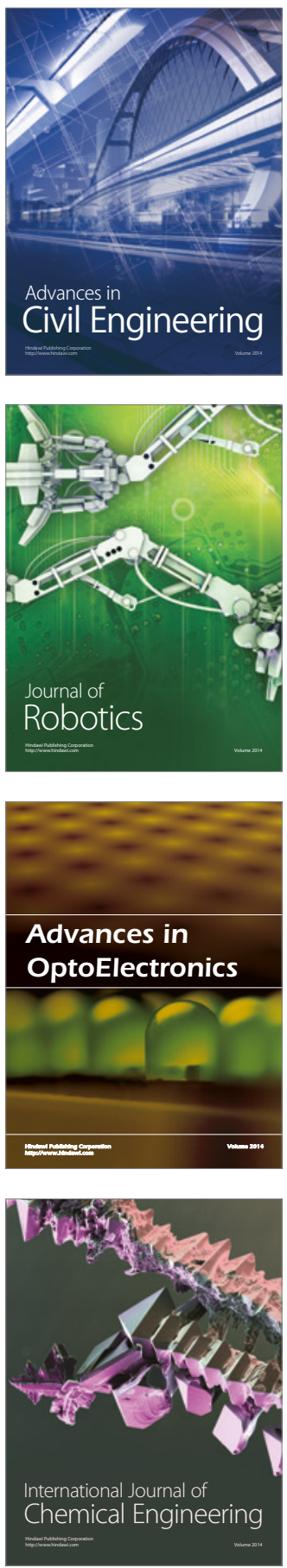

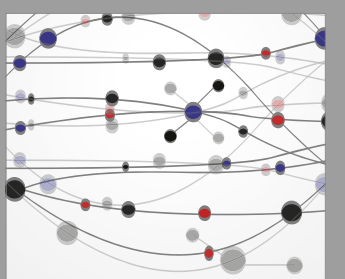

The Scientific World Journal

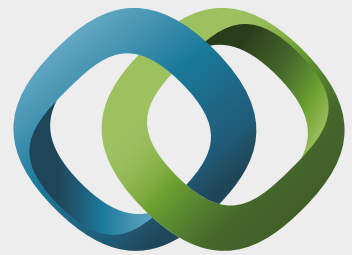

\section{Hindawi}

Submit your manuscripts at

https://www.hindawi.com
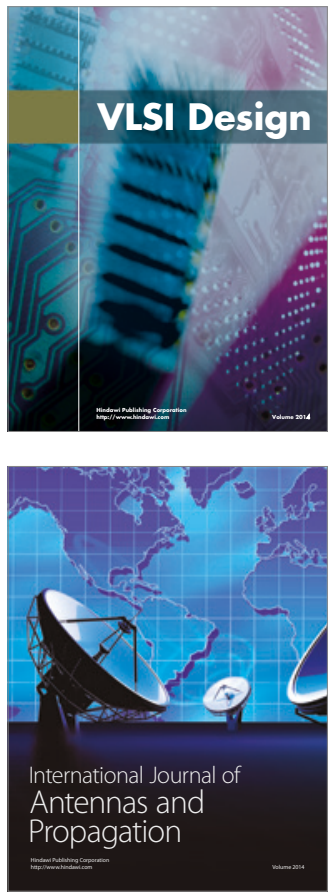

\section{Rotating}

Machinery
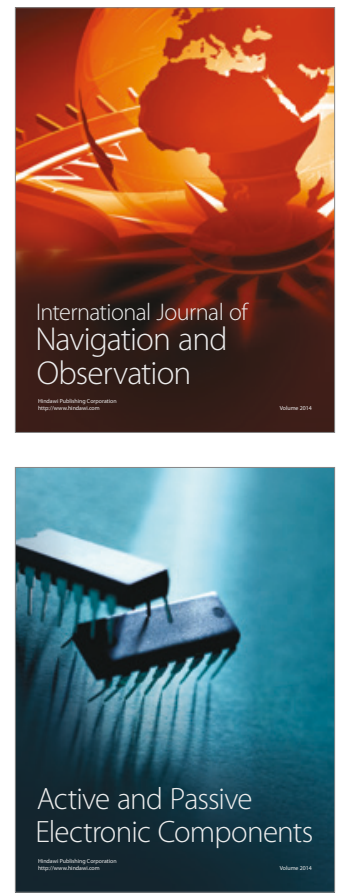
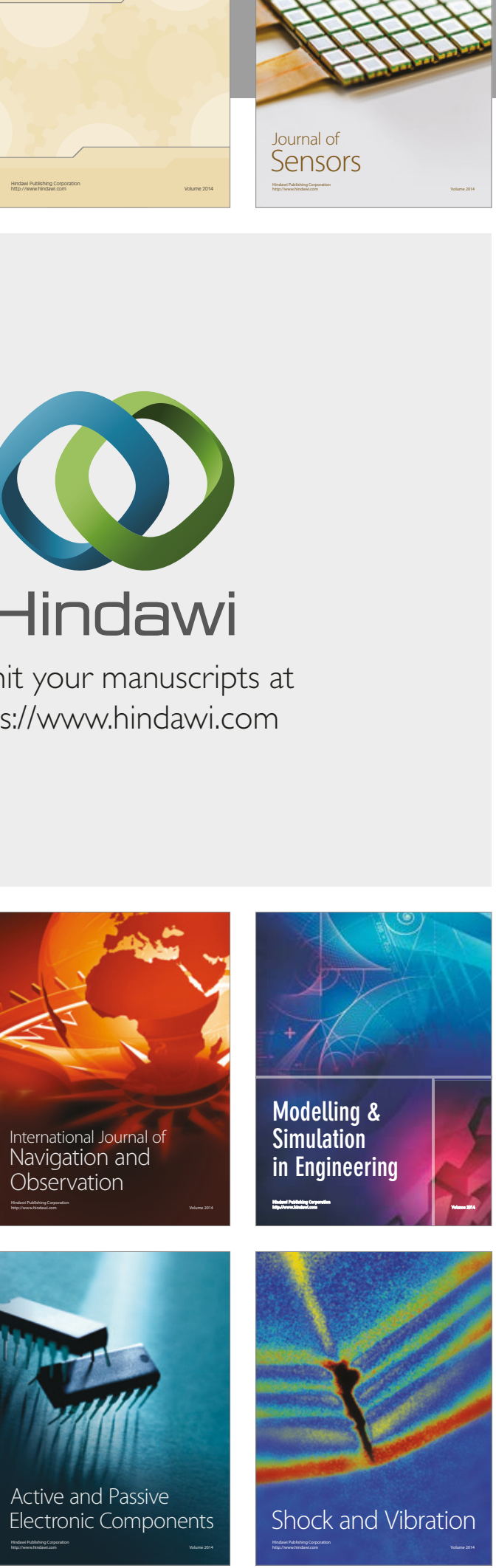
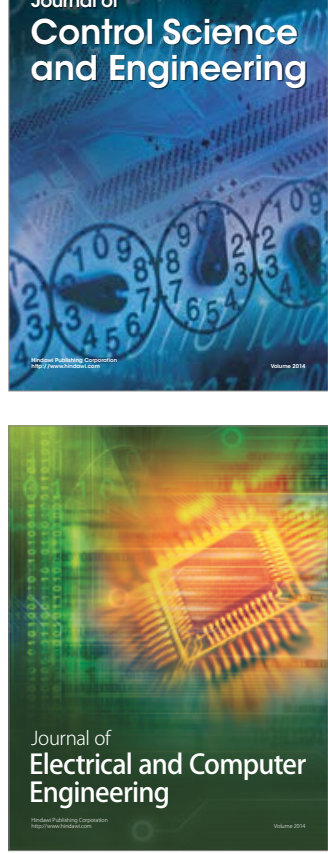

Distributed

Journal of

Control Science

and Engineering
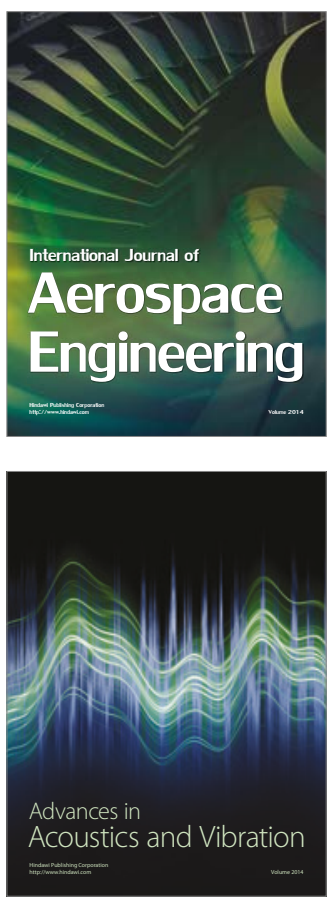

Sensor Networks 